\title{
Reply to Fleming: Symmetries, Observables, and the Occurrence of Events
}

\author{
Thomas Pashby
}

October 3, 2013

First I want to thank Prof. Fleming for his detailed, thoughtful, and thought-provoking remarks, and particularly for his generous advice on how to improve successive versions of the conference paper presented here in its final form. While our continuing correspondence has led to something of a convergence of views, there are several differences of opinion that resist this reconciliation. In this reply I will present my side of two of these remaining disputes, which are particularly relevant for understanding how accepting my account of event time observables (sketched in the final section of the paper) leads to a distinctive view of quantum theory. The core of what is distinctive about my view is that it restores a certain symmetry between time and space with respect to what is observable according to the theory. The empirical motivation for this view is that often the outcomes of an experiment may be located in time as well as in space. The theoretical consequence of this view is that time covariant POVMs must be provided which have this specific empirical interpretation.

\section{On What is Located Where, and Whence}

I think that both Prof. Fleming and I agree that 'standard QM' (i.e. the Dirac-von Neumann formalism) is ill-equipped to supply observables that apply not to an instant of time but an interval of time. However, whereas I view that as a problem to be overcome through (conservative) modification of the formalism, Prof. Fleming appears to view this feature as somehow constitutive of quantum theory, writing:

QM is a theory of temporally persistent dynamical systems, indeed of eternal systems which live in a fixed classical space-time. 
... The basic observables of standard QM ... are designed to answer questions about ... possible properties of persistent physical systems at specified times (or, relativistically, on specified spacelike hypersurfaces). (His emphasis.)

On the other hand, I take the view that the time-dependent observables of QM (and in particular position observables) should be thought to concern the properties of events in whose production the system is involved, not the properties of the system itself.

In order to draw out this contrast, let us consider the instantaneous measurement of position of a system in state $\psi$ at a particular time $t$, corresponding to the Heisenberg picture projection $P_{\Delta}(t)=U_{t} P_{\Delta} U_{t}$. For Prof. Fleming, the (pure) state $\psi$ describes the (possible) properties of an eternally persisting object, whose values are realized on measurement (according to the conventional interpretation) or (according to the dynamical collapse interpretation he favors) given (nearly) determinate values by a distinct stochastic localization process. ${ }^{1}$ Regardless, what is being localized (and what is correspondingly measured by $P_{\Delta}(t)$ ) is a property of the system as a whole, namely its location. According to his view, if we have a situation in which $\left\langle\psi \mid P_{\Delta}(t) \psi\right\rangle=1$, then this reveals the quantum system described by $\psi$ to be entirely located within the spatial region $\Delta$ at time $t^{2}$ This view leads to certain difficulties regarding relativistic localization, to which much of Prof. Fleming's work has been addressed.

In contrast, my view is that, ultimately, $P_{\Delta}(t)$ is to be understood in terms of the location of a detection event within a suitable experimental apparatus. (Think here of a diffraction experiment with a luminescent screen or a Wilson cloud chamber.) Thus $\left\langle\psi \mid P_{\Delta}(t) \psi\right\rangle=1$ is to be interpreted as saying, with certainty, that such an event did occur located within $\Delta$ at time $t$. On this view, $P_{\Delta}(t)$ does not concern the location of the system entire, conceived as a persisting material object, but rather the experimentally determinable, spatio-temporally located events that occur in interaction of the system in question with other physical systems, namely (in this case) the experimental apparatus. ${ }^{3}$ We can call this view the event-first view of quantum theory, in which the quantum state concerns the probabilities for the

\footnotetext{
${ }^{1}$ For some potential difficulties of thinking about quantum systems as persisting objects, see my 'Do Quantum Objects Have Temporal Parts?,' Philosophy of Science (forthcoming).

${ }^{2}$ Note that this is precisely the interpretation adopted by Wightman (1962).

${ }^{3}$ To head off potential confusion, I do not mean to say that quantum theory concerns only such events, or that the events in question need to be observable (in the sense of van Fraassen, i.e., by human senses alone).
} 
occurrence of events, in contrast to the system-first view that Prof. Fleming adopts, in which the quantum state concerns the (potential) properties of a persisting object.

In this connection, let me quote Dirac's remarks to the 1927 Solvay conference regarding the interpretation of quantum theory, in which he famously claimed that 'Nature makes a choice.' (It is important to note that Dirac is here speaking before the establishment of "standard QM," i.e. in advance of Hilbert space methods having become commonplace.)

It is essential that the result of an experiment shall be a permanent record. The numbers that describe such a result must help to not only describe the state of the world at the instant the experiment is ended, but also help to describe the state of the world at any subsequent time. These numbers describe what is common to all the events in a certain chain of causally connected events, extending indefinitely into the future. Take as an example a Wilson cloud expansion experiment. The causal chain here consists of the formation of drops of water round ions, the scattering of light by these drops of water, and the action of this light on a photographic plate, where it leaves a permanent record. The numbers that form the result of the experiment describe all of the events in this chain equally well and help to describe the state of the world at any time after the chain began.

(Bacciagaluppi \& Valentini, 2009, p. 447)

What do these 'numbers' (i.e. $c$-numbers) concern? I contend that they concern (at least) the spatio-temporal location of the ionization event (brought about by the quantum system in question) that set in motion the chain of events leading to a permanent record of this outcome. ${ }^{4}$ On the events-first view, the quantum state is to provide predictions of where these events are located in space and in time.

Now, if the outcomes of this experiment are to be predicted by observables of the theory, then these observables had better assign probabilities to the spatio-temporal location of these events. As I discussed in the paper, it is hard to see how standard QM could do this if $P_{\Delta}(t)$ is interpreted as supplying probabilities for the results of a measurement at time $t$. But Prof.

\footnotetext{
${ }^{4}$ While I do think that Dirac's words here present an effective characterization of the view I am advocating, his language of 'causal connections' should not be read as an invocation of any philosophically-loaded notion of causation. Given Dirac's self-described contempt for philosophy, and generally instrumentalist bent, it is anyway unlikely that he had such ideas in mind.
} 
Fleming suggests an alternative view of quantum theory, which differs from the usual Schrödinger dynamics by the addition of an additional dynamical process of stochastic state reduction. On this view, presumably, the appearance of particle (or quanton) tracks in a cloud chamber (which, note, are not continuous trajectories but a discontinuous series of sites of condensation) is due to the repeated localization of the system (e.g. an alpha particle) and its subsequent interaction with the water molecules; or, perhaps, the spontaneous localization of both water molecules and alpha particle brought about by the stochastic localization process. Prof. Fleming notes that these spontaneous localizations are, like measurement in standard QM, to be thought of as occurring instantaneously and at definite times. This leads to the view that,

... there would be no question of measuring when the primordial reductions occur and trying to measure just when a measurement exploited reduction measurement exploited reduction occurs (within the exploiting measurement) would be an instance of measuring a case specific time observable.

However, on the event-first view, the theory already provides probabilities for events to occur at particular times in particular locations (at least, when supplied with appropriate observables), and so there is no need to introduce an additional mechanism to bring about their occurrence. On the eventfirst view, the spatio-temporal properties measured in an experiment are not the properties of the system since it is the events that come to be spatio-temporally located not 'the system.'

Admittedly, the interpretation of event time POVMs in terms of conditional probabilities for an event to occur at a particular time (given that the event occur at some time) is not inconsistent with the idea that the event in question is a "primodial reduction." However, the probabilities supplied by a dynamical collapse model would not (in general) meet the required condition. ${ }^{5}$ And since these probabilities may be determined from the Schrödinger equation (or the extended Schrödinger equation) without introducing an additional stochastic process, there is simply no technical, nor interpretative, need for such a process. That is, we need not imagine that in, e.g., a cloud chamber ionization two separate events occur: first the state reduction and then the ionization. The event-first view maintains that

\footnotetext{
${ }^{5}$ It may be objected that it is only under special circumstances that such probabilities apply, but note that experimental arrangements are often chosen so as to expressly provide these circumstances.
} 
in reality there is a single thing that occurs at some time in a particular spatial location: the ionization event (which is followed by certain other events which together comprise the collection of water molecules observed by the human eye).

I conclude this section by addressing Prof. Fleming's skepticism regarding the experimental realization of the time of arrival operator that we both consider. He complains that the statistics of such an operator concern arrival at a point (or plane) and require an experiment to run for all (eternal) time. An exactly analogous objection could equally be mounted to the instantaneous measurement of the position observable: the statistics it supplies concern an experiment that takes place over all of (infinite) space, and which runs for a mere instant. Regarded as a description of an experiment that takes place in a (spatially bounded) lab over some extended period of time, we might equally call the position observable a "toy model too silly for words."

However, in neither case do I think the idealizations involved are particularly problematic, and we may allow the respective operators to provide an (admittedly idealized) description of an experiment that could be performed and repeated in a lab. Just as we do not think the position observable fails to apply to such experiments due to the practical difficulties involved in constructing infinitely large detectors that are sensitive at an instant, so we should not think the time of arrival to be without experimental content in the absence of detectors stretching across all of space, sensitive for the entire history of the universe. Indeed, few diffraction experiments end without multiple successful detections at a screen of limited (but finite) spatial extent, sensitive over an extended period of time.

\section{On the Underdetermination of POVMs}

In the immediately preceding discussion of the time of arrival operator $T_{a}$, I glossed over an important point: the measurement statistics for the time of arrival are not provided by the time of arrival operator, but rather the POVM (uniquely) associated with it. This is no less the case for position since specifying the self-adjoint operator $Q$ also serves to uniquely determine a POVM, but in this case the POVM happens to be in addition a PVM. Without yet going into the details, this is because, although $Q$ and $T_{a}$ are each symmetric, only $Q$ is self-adjoint. ${ }^{6}$ This association of $Q$ with a PVM rather than a POVM is thus guaranteed by the Spectral Theorem. I take

\footnotetext{
${ }^{6} \mathrm{~A}$ bounded operator $A$ is symmetric iff $\langle\phi \mid A \psi\rangle=\langle A \phi \mid \psi\rangle$ for all $\psi, \phi \in \mathcal{H}$.
} 
Prof. Fleming's worries, expressed in his $\S 5$, to be directed at the use of POVMs that are not PVMs, and I take his major worry to be this: in moving beyond the usual association of self-adjoint operators with observables of the theory we break the one-to-one correspondence of operators with PVMs, and so run the risk of (i) underdetermation of the POVM by the specification of a particular operator, and so (ii) vesting the mere mathematical definition of a POVM with undeserved physical significance.

First, let me clear up a terminological distinction that, although present in Prof. Fleming's explication, is not given sufficient emphasis. In this context, a Positive Operator Valued Measure is essentially a map from a collection of measurable subsets of $\mathbb{R}$ to a set of positive operators on a Hilbert space $\mathcal{H}{ }^{7}$ Nowhere does Prof. Fleming explicitly define such a map. He chooses instead to consider what are known as generalized resolutions of the identity, his (12). ${ }^{8}$ Now, the relationship between these and the associated POVM is very close (as he indicates): if $\hat{P}(x)$ is a generalized resolution of the identity and $\Delta$ is a measurable subset of $\mathbb{R}$ (an open set of points $x$ dense in some interval) then

$$
E_{\Delta}=\int_{\Delta} d \hat{P}(x)
$$

is the positive operator associated with $\Delta$, and $\Delta \mapsto E_{\Delta}$ is the POVM defined by $\hat{P}(x)$.

What Prof. Fleming calls the "broad approach" is, I believe, fairly standard in the literature he discusses, and takes as its starting point the assumption that the POVM $\Delta \mapsto E_{\Delta}$ provides all the relevant experimental information. Adherents of the broad approach would, therefore, prefer to pick out the POVM corresponding to a particular physical parameter or experimental situation uniquely by means of physically motivated symmetry requirements. The association of a POVM with a symmetric operator via its first moment is thus of lesser importance, and one would not necessarily need to consider such an operator in using the POVM to provide the statistics for a given measurement situation. I would not call this the broad approach, but rather the POVM-first approach, in contrast to the operator-first approach that Prof. Fleming appears to prefer. ${ }^{9}$

\footnotetext{
${ }^{7}$ Positive operators $E$ have a real spectrum and have the property that $\langle\phi \mid E \phi\rangle \geq 0$ for all $\phi \in \mathcal{H}$. A projection $P^{2}=P$, with spectrum $\{0,1\}$, is obviously a positive operator.

${ }^{8}$ See, e.g., Alkhiezer \& Glazman (1993, p. 121).

${ }^{9}$ Note that an adherent of the POVM first approach need not be committed to the use of POVMs that are not also PVMs (and thus uniquely associated with self-adjoint operators) - she could still maintain that it is just those POVMs that are PVMs which have physical significance.
} 
To give a concrete example, consider Wightman's (1962) classic analysis of localization. Rather than defining a self-adjoint position operator, Wightman assumes the existence of a PVM whose assignment of projections to spatial regions covaries with translations under a unitary representation of the Euclidean group. The major achievement of the paper is the proof that a PVM is uniquely defined by this requirement (along with some other symmetry and regularity conditions) in both Galilei and Poincaré invariant quantum theory. Having done so, we are able to see that the self-adjoint operators that these PVMs define are none other than the usual position operator (or the Newton-Wigner operator in the relativistic case). But Wightman's physical interpretation is given in terms of the PVM: in this case $P_{\Delta}$ supplies the probability that the system is found to be located in the region $\Delta .^{10}$ In posing this 'experimental question' it is not the position observable $Q$ that is measured but the projection $P_{\Delta}$. So already, without going beyond the set of PVMs, there may be good reasons to view the POVM as more physically significant than its first moment.

The reason it is necessary to go beyond PVMs when defining case specific time POVMs is that the symmetry that one expects such POVMs to respect - time translation covariance - cannot be obeyed by a PVM (this is implied by 'Pauli's theorem'). It is for this reason that one must go beyond the set of PVMs in defining probabilities for the occurrence of events in time. From this perspective - according to which the POVM takes center stage - the failure of the second moment of the POVM to equal the square of time operator (Fleming's (19)) only serves to indicate that the time operator is of minor interest, and one's attention is best devoted to the underlying POVM. From this perspective, there is no need to worry about the failure of a (non-maximally) symmetric operator to uniquely determine a POVM since one already has the POVM in hand, which was picked out independently by symmetry requirements. From this perspective, there is no problem of underdetermination of POVM by symmetric operator, since there is no need to determine the POVM from the symmetric operator: instead, the symmetric operator is picked out by the POVM. Moreover, as Fleming acknowledges, the underdetermination problem he raises through his equation (20) does not, according to his (21), lead to any empirical difference: what is significant is that $T_{a}$ is the first moment of the relevant POVM; the fact that it is also the first moment of other POVMs is irrelevant.

Nonetheless, let us consider the problem from the 'narrow' perspective

\footnotetext{
${ }^{10}$ In the previous section I suggested that $P_{\Delta}$ is better conceived as concerning the location of a detection event rather than the entire system.
} 
that begins with a symmetric operator and seeks the associated POVM. Every POVM specifies a symmetric operator on $\mathcal{H}$ as its first moment (Werner, 1986, Prop. 3, p. 796). ${ }^{11}$ Given a symmetric operator $S$ on $\mathcal{H}$ there are (generally speaking) three possibilities: either $S$ has a unique self-adjoint extension (it is essentially self-adjoint), or $S$ has many self-adjoint extensions, or $S$ has no self-adjoint extension (it is thus maximally symmetric). ${ }^{12}$ In the first case, $S$ corresponds to a unique PVM. In the second case, $S$ corresponds non-uniquely to many PVMs. In the third case, $S$ corresponds uniquely to a POVM (as Prof. Fleming acknowledges). Thus it is unclear how the underdetermination worry (regarding POVMs, remember) is going to get traction: according to this classification, there is only underdetermination in the second case, which involves a non-uniqueness of PVMs, not POVMs. ${ }^{13}$

\section{References}

Earman, J. (2009). Essential self-adjointness: implications for determinism and the classical-quantum correspondence. Synthese, 169(1), 27-50.

Gilbert, R. C. (1977). The deficiency index of a symmetric ordinary differential operator with complex coefficients. Journal of Differential Equations, $25(3), 425-459$.

Riesz, F. \& Sz.-Nagy, B. (1990). Functional analysis. New York: Dover.

\footnotetext{
${ }^{11}$ Note that, as with the Spectral Theorem, we are specifically considering here a map from the Borel sets of the real line.

${ }^{12}$ This classification corresponds to $S$ having (i) deficiency indices $(0,0)$; (ii) deficiency indices $(n, n)$; (iii) deficiency indices $(n, 0)$ or $(0, n)$. See (e.g.) (Riesz \& Sz.-Nagy, 1990, p. $328)$. It is possible to construct a symmetric operator that lies outside this classification, i.e., with deficiency indices $(n, m)$ such that $n \neq m, m, n \neq 0$. These are known to arise in considering third-order complex differential operators (Gilbert, 1977). Since the motivation was to find operators that are closely resemble the usual observables of quantum theory I think we can safely exclude this case.

${ }^{13}$ See Earman (2009) for a recent philosophical analysis of non-essentially self-adjoint Hamiltonians.
} 